\title{
Editorial
}

\section{T N Guru Row and Angshuman Roy Choudhury, Guest Editors}

The last issue of Resonance for the year 2014 is aptly dedicated to the celebration of the International Year of Crystallography. At the opening ceremony on January 20,2014, which was held in Paris, the Secretary General of the United Nations stated, "It is at the very core of revealing the structure of DNA, fabricating computer memories and designing potent new drugs, but only a tiny fraction of the world's people is aware of the science of crystallography, a knowledge gap the United Nations hopes to erase this year."

On January 30, 2014, the Department of Posts, India, issued a stamp at the Indian Institute of Science, Bangalore, to commemorate the achievements of crystallographers across the globe and highlight the importance of this field to young scientists and the general public. This stamp (shown on the cover page) depicts a diamond and the structure of curcumin, the active constituent of turmeric, as determined by X-ray crystallography. The diamond is known for its exceptional hardness and the flashes of light given off by its natural crystal structure. Curcumin is the compound responsible for the bright orange of turmeric which exhibits antioxidant, anti-inflammatory, antimicrobial and anticarcinogenic properties. It is interesting that the structure of curcumin was established at the Solid State and Structural Chemistry Unit at IISc, Bangalore.

The celebrations continued throughout the year with a large number of universities arranging special one-day meetings and workshops. The 42nd National Seminar on Crystallography held in Delhi during November 21-23 was an occasion to herald IYCr 2014 in India. To celebrate IYCr in a wider context, there were three other national seminars on crystallography in $2014-$ NSC43A: IISER, Mohali (Convener: A Roy Choudhuri); NSC43B: 
Sardar Patel University, Vallabh Vidyanagar (Convener: Urmila Patel) and NSC43C: CSIRCentral Drug Research Institute, Lucknow (Convener: R Ravishankar). The 6th International Symposium on Recent Trends in Macromolecular Structure and Function (ISRTMSF-2014; Convener: D Velmurugan), was held from January 22-14, 2014 at Chennai. An International Workshop on Symmetry Relationships between Crystal Structures with Applications to Structural and Magnetic Phase Transitions was held at Banaras Hindu University from October 27-31, 2014 (Convener: D Pandey).

In order to popularize crystallography, lectures by eminent crystallographers were held in remote areas of the country. Articles in vernacular magazines/newspapers have been circulated among schools and colleges to provide a glimpse of crystallography to non-practitioners.

When Resonance asked us to guest edit the December issue, we were faced with an uphill task as most of the crystallographers were involved in multifarious activities, and with a lot of luck we were able to get excellent articles for this issue from several leading crystallographers of the country. We thank all the authors for their efforts to make this a very useful handbook for the readers of Resonance.

It is also heartening to note that we were able to cover most of the current cutting edge areas in crystallography pursued by several young scientists. The readers will relish the broad coverage we could achieve starting from the history of crystallography, getting answers to queries like why do we need structures determined, and why crystallography is indeed the gold standard for structure determination. We have articles covering methodologies like cryocrystallography, inputs for drug design from macromolecular crystallography, framework structures and combined X-ray and cryo-electron microscopy for large structures. We believe that the collection of articles provides a current status overview of the fascinating area of X-ray crystallography initiated a hundred years ago with the structure of 'common salt' by W $\mathrm{H}$ Bragg and W L Bragg!

Finally, a word on the excellent cartoon by Ayan Guha, which depicts the discovery and growth of X-ray diffraction as symbolically representing the true spirit of X-ray crystallography. We thank him for his efforts and creativity.

T N Guru Row* and Angshuman Roy Choudhury** *Solid State and Structural Chemistry Unit, IISc, Bangalore **Department of Chemical Sciences, IISER, Mohali,Punjab. 